\title{
Exposure to nitric oxide drives transition to resuscitation-promoting factor- dependency in mycobacteria
}

Sarah M. Glenn ${ }^{1, \dagger}$, Brindha Gap-Gaupool ${ }^{1 \dagger}$, Simon J. Waddell ${ }^{2}$, Joanna Bacon ${ }^{3}$, Marialuisa Crosatti ${ }^{1}$, Jennifer Hincks ${ }^{4}$, Sharon L. Kendall ${ }^{5}$, Olga Riabova ${ }^{6}$, Natalia Monakhova $^{6}$, Jeffrey Green ${ }^{7}$, Obolbek Turapov ${ }^{1}$, Vadim Makarov6, Galina V. Mukamolova $^{1 \$}$

tThese authors contributed equally to the manuscript

${ }^{1}$ Leicester Tuberculosis Research Group, Department of Respiratory Sciences, University of Leicester, Leicester, LE1 9HN UK; ${ }^{2}$ Global Health and Infection, Brighton and Sussex Medical School, University of Sussex, Brighton, BN1 9PX, UK; ${ }^{3}$ TB Discovery Group, National Infection service, Public Health England, Porton Down, UK SP4 0JG; ${ }^{4}$ FACS Facility Core Biotechnology Services, University of Leicester, Leicester, UK; ${ }^{5}$ Centre for Endemic, Emerging and Exotic Disease, The Royal Veterinary College, Hatfield, Hertfordshire, AL9 7TA, ${ }^{6}$ Research Center of Biochemistry, Russian Academy of Sciences, Moscow, Russia; ${ }^{7}$ Molecular Biology and Biotechnology, University of Sheffield, Sheffield, S10 2TN, UK.

\section{Source of funding}

This work was supported by the Biotechnology and Biological Sciences Research Council (BBSRC), grant numbers BB/K000330/1 and BB/P001513/1 (GVM, JG), the Russian Science Foundation under grant 21-15-00042 (VM, NM, OR), Innovative Medicines Initiative Joint Undertaking under grant agreement 115337, the resources of which are composed of financial contributions from the European Union's Seventh Framework Program (FP7/2007-2013) and EFPIA companies' in-kind contributions (SJW and GVM). BGG was funded by Midlands Integrative Biosciences Training 
Partnership (MIBTP) funded by BBSRC (grant number BB/M01116X/1) and the

Public Health England PhD-studentship fund.

\section{${ }^{\$}$ Corresponding author}

Galina V. Mukamolova, Leicester Tuberculosis Research Group, Department of Respiratory Sciences, University of Leicester, Leicester, LE1 9HN UK

Phone: +44 (0) 116229 7053, email: gvm4@leicester.ac.uk

\section{Abbreviations}

BCG - Bacillus Calmette-Guérin

$\mathrm{CC}-$ control compound

CFU - colony forming unit

CSN - culture supernatant

DAF-FM diacetate - 4-amino-5-methylamino-2',7'-difluorofluorescein diacetate

DCB - differentially culturable bacilli

MPN - most probable number

Mtb - Mycobacterium tuberculosis

$\mathrm{NO}$ - nitric oxide

ND - nitric oxide donor

PMA - phorbol myristate acetate

$\mathrm{PI}$ - propidium iodide

Rpf - Resuscitation-promoting factor

TFOE - Transcription Factor Over Expression

TB - tuberculosis

\section{Author's contributions}


Conception and design of the study: GVM, VM, SMG, BGG, SJW, JG. Data acquisition: SMG, BGG, SJW, MC, JH, OR, NM, GVM, OT. Data analysis and interpretation: all authors. Generation of essential materials and reagents: SLK, JB, NM, OR, VM. Manuscript drafting or critical revision: all authors. Final approval of the submitted version: all authors.

\section{Running title}

Nitric oxide induces Rpf-dependency

\section{ABSTRACT}

Rationale: Mycobacterium tuberculosis (Mtb) has a devastating impact on global health. Mtb is the causative agent of human pulmonary tuberculosis (TB), which claims 1.4 million lives per annum. During infection, differentially culturable bacilli (DCB) that fail to produce colonies on solid media but grow in liquid media supplemented with resuscitation-promoting factor (Rpf) proteins are formed. These Rpf-dependent mycobacteria are a major barrier to developing improved TB treatments and diagnostic tools.

Objectives: To evaluate whether exposure to nitric oxide (NO), a prominent host defense molecule, drives the generation of Rpf-dependent DCB.

Methods: A new NO donor (ND) for sustained NO release was synthesized. Growth assays, flow cytometry analysis, and global transcriptional profiling, were used to characterize ND-treated Mtb and Mycobacterium bovis BCG in vitro. Mycobacterial DCB phenotypes were also investigated after infection of THP-1 human cells, which were activated with retinoic acid and vitamin $\mathrm{D}_{3}$. 
Measurements and Main Results: DCB were generated after exposure to ND and during infection of activated THP-1 cells. Resuscitation of these DCB was severely impaired by a specific Rpf inhibitor (3-nitro-4-thiocyanato-phenyl)-phenyl-methanone. Global transcriptomic analyses revealed the down-regulation of three ( $r p f A, r p f B$ and rpfE) of the five genes coding for Rpf proteins.

Conclusion: A new model for investigation of the transition of Mtb into an Rpf dependent differentially culturable state is presented. The system reveals mechanistic insights into the generation of Rpf-dependent Mtb during TB disease and demonstrates the role of $\mathrm{NO}$ in this process.

Word count: 238

Key words: Tuberculosis, Mycobacterium tuberculosis, differentially culturable bacilli, Resuscitation-promoting factor, nitric oxide

\section{INTRODUCTION}

Mycobacterium tuberculosis (Mtb) is the causative agent of tuberculosis (TB), which resulted in the death of 1.4 million people in 2019 [1]. Standard TB treatment lasts at least 6 months and significant resources are directed towards developing shorter and more effective treatments [2]. One barrier to achieving this goal is the generation of tubercule bacilli populations during infection that are not detectable by standard tools [3]. These bacilli do not produce colonies on agar and only grow in liquid medium, often require supplementation with culture supernatant (CSN) $[3,4]$ and therefore called differentially culturable bacilli (DCB) [4]. In published literature they have been also defined as non-culturable cells [5], viable but non-culturable bacilli [6] and differentially detectable cells [7]. DCB have higher tolerance to some anti-TB drugs [3, 
8], and drug treatment may increase the proportion of DCB in patients $[3,7,9,10]$. These cells are believed to be in a special dormant state that requires resuscitation in liquid media or CSN, however, DCC are currently poorly characterised.

CSN contains resuscitation-promoting factor (Rpf) proteins, which are peptidoglycan remodeling enzymes [11] that are able to resuscitate DCB from the sputum of TB patients [3, 12] and animal lungs [13]. While resuscitation of these Rpfdependent Mtb has been linked to TB relapse in mice [14], the importance of this process in human TB remains to be established.

The molecular mechanism of DCB generation is currently unknown, although the in vivo environment has been proposed as a key factor [13]. Nitric oxide (NO) is a wellcharacterized component of the innate immune system that is deployed to control intracellular pathogens [15]. While its precise role in controlling human TB is unclear, the inducible NO synthase (iNOS) of macrophages is critical for controlling TB in mice [16] and humans [reviewed in 17]. NO may limit Mtb intracellular growth due to its direct bactericidal effects or by activation of inflammatory cytokines [17] and interference with granulocytic inflammation [18]. Compounds that release NO (NO donors) produce a range of outcomes from bacteriostatic to bactericidal when added to Mtb cultures and dependent in part on the stability and sustained release of NO from the donor [17].

Here, it is shown that sustained exposure to a novel NO donor (ND) rapidly generated DCB of Mtb and Mycobacterium bovis (BCG). Resuscitation of these DCB in liquid medium, measured by Most Probable Number (MPN) assay was abolished by a specific Rpf inhibitor 3-nitro-4-thiocyanato-phenyl)-phenyl-methanone [13, 19], suggesting that NO exposure drives Rpf-dependency. Transcriptomic analysis revealed that ND dramatically down-regulated expression of three of the five genes 
coding for Rpf proteins (rpfA, rpfB and rpfE). Importantly, NO activation of THP-1 macrophages with retinoic acid and vitamin $D_{3}$ promoted NO production and formation of DCB. Taken together, these observations suggest that NO plays a role in generation of DCB during TB infection.

\section{METHODS}

\section{Organisms and media}

Liquid cultures were grown at $37^{\circ} \mathrm{C}$ with shaking (100 rpm) for Mtb and static for $M$. bovis BCG Glaxo in Middlebrook $7 \mathrm{H} 9$ broth, supplemented with $0.2 \%$ (v/v) glycerol, 10\% (v/v) albumin-dextrose-catalase (ADC), and 0.05\% (w/v) Tween 80 (hereafter 7H9 medium). Culture supernatants (CSN) were prepared from exponentially growing cultures $\left(O D_{580 n m}\right.$ of $\left.0.6-0.8\right)$ and sterilized by double filtration $(0.22 \mu \mathrm{m}$ filters $)$. For MPN assays, $50 \%$ sterile CSN was mixed with $7 \mathrm{H} 9$ medium. The Rpf inhibitor (3-nitro4-thiocyanato-phenyl)-phenyl-methanone [19] was added to a final concentration of $35 \mu \mathrm{M}$. The dosR mutant and complemented strain have been previously described [20]. M. bovis BCG stocks for macrophage infection were prepared from exponentially growing cultures $\left(\mathrm{OD}_{580 \mathrm{~nm}} \sim 0.4-0.5\right)$. THP-1 human monocyte cell line (ATCC® TIB$202^{\mathrm{TM}}$ ) was grown at $37^{\circ} \mathrm{C}$ with $5 \% \mathrm{CO}_{2}$ in Roswell Park Memorial Institute (RPMI) 1640 medium supplemented with $10 \%(\mathrm{v} / \mathrm{v})$ heat inactivated fetal bovine serum.

\section{Assessment of viable counts}

MPN counts were quantified as previously described $[3,8]$. Briefly, sealed 48-well plates with serially diluted mycobacteria in 7H9 medium (MPN_7H9) or 7H9 medium supplemented with $50 \%(\mathrm{v} / \mathrm{v})$ culture supernatant (MPN_CSN) were incubated at $37^{\circ} \mathrm{C}$ for 8 weeks. The MPN calculator program was used for determination of MPN counts [21]. For CFU counts, serially diluted samples were spotted onto Middlebrook $7 \mathrm{H} 10$ 
agar plates supplemented with $10 \%(\mathrm{v} / \mathrm{v}) \mathrm{ADC}$ and $0.5 \%$ glycerol and incubated at $37^{\circ} \mathrm{C}$ for up to 8 weeks. Resuscitation Index (RI) was defined as $\mathrm{RI}=\log _{10} \mathrm{MPN}-\log _{10}$ CFU. Limits of detection for CFU and MPN counts were 24 CFU $/ \mathrm{ml}$ and 4.6 cells $/ \mathrm{ml}$, respectively.

Synthesis of NO donor (ND) and control compound (CC) and quantification of NO release

NO donor 3-cyano-5-nitropyridin-2-yl diethyldithiocarbamate (ND) and Control compound 3-cyano-4,6-dimethyl-5-nitropyridin-2-yl piperidine-1-carbodithioate (CC) were synthesized as described in the Online Supplement. The concentration of NO generated by ND and CC were quantified using Griess reagents as previously described [22]. ND or CC were incubated in $200 \mathrm{mM}$ sodium phosphate buffer $(\mathrm{pH}$ 6.0) at a final concentration of $200 \mu \mathrm{M}$ for 24 hours and absorbance at $540 \mathrm{~nm}$ was measured at different time points. Known concentrations of sodium nitrite were used to construct a calibration curve.

\section{Treatment of Mtb with ND and CC}

Mtb was grown in $7 \mathrm{H} 9$ medium to $\mathrm{OD}_{580 \mathrm{~nm}}$ of $0.8-1.0$. Cells were diluted with fresh $7 \mathrm{H} 9$ medium to an $\mathrm{OD}_{580 \mathrm{~nm}}$ of 0.1 in a total volume of $5 \mathrm{ml}$. ND or CC were dissolved in DMSO and added to cultures to a final concentration of $100 \mu \mathrm{M}$. Cultures were incubated at $37^{\circ} \mathrm{C}$ without shaking for 24 hours. In other experiments, mycobacteria were treated with $200 \mu \mathrm{M}$ spermine-NONOate (equivalent to up to $400 \mu \mathrm{M} N O$; Sigma), or $5 \mathrm{mM} \mathrm{NaNO}_{2}$.

\section{Flow cytometry staining and analysis}

For detection of NO in ND-treated M. bovis BCG, DAF-FM diacetate (4-amino-5methylamino-2', $7^{\prime}$-difluorofluorescein; ThermoFisher) at concentration of $5 \mu \mathrm{M}$ was used [23]. Stained bacteria were analysed in CytoFLEX flow cytometer using filters 
for excitation $495 \mathrm{~nm}$ and emission $515 \mathrm{~nm}$ and CytExpert software (Beckman Coulter).

LIVE/DEAD BacLight (ThermoFisher) stained $M$. bovis BCG were studied in FACSAria flow cytometer using BD FACSAria ${ }^{\mathrm{TM}}$ software (BD Bioscience). The following parameters were applied: SYTO 9 excitation/emission at 480/500 nm; PI excitation/emission at 490/635 nm; 10,000 events were recorded from each sample. Single parameter fluorescence overlay plots and dual parameter dot plots were generated as previously described [24]. The gating strategy is summarized in the Online Supplement. Heat-killed M. bovis BCG were included as a control.

\section{Transcriptomic analyses}

Three biological replicates of RNA from Mtb were extracted 4 hours after treatment with either ND or CC using the GTC/Trizol method [25]. RNA (2 $\mu \mathrm{g})$ was labeled with Cy3 fluorophore and hybridized to a Mtb microarray (ArrayExpress accession number ABUGS-41) as previously described [26]. Fully annotated microarray data are available in ArrayExpress (E-MTAB-10776).

For quantitative RT-PCR, RNA was reverse transcribed to cDNA using SuperScript II reverse transcriptase kit (ThermoFisher Scientific) with mycobacterial genome-directed primers [27]. The qPCRs were run in the Rotor-gene 600 instrument (Corbett Research) using the $2 \times$ SYBR green master mix (ThermoFisher Scientific) and primers (Table S1). Levels of expression were normalized to $16 \mathrm{~S}$ rRNA.

\section{Macrophage infection}

Human THP-1 monocytes were seeded at $2 \times 10^{5}$ cells $/ \mathrm{ml}$ in 24 well plates (Greiner) followed by activation either with retinoic acid $(1 \mu \mathrm{M})$ and vitamin $D_{3} /$ cholecalciferol (1 $\mu \mathrm{M}$ ) or $10 \mathrm{ng} / \mathrm{ml}$ PMA (phorbol myristate acetate) for 72 hours. The macrophages were infected with $M$. bovis BCG at an $\mathrm{MOI}$ of 0.5 and incubated at $37^{\circ} \mathrm{C}$ with $5 \% \mathrm{CO}_{2}$ for 3 
hours, followed by treatment with $200 \mu \mathrm{g} / \mathrm{ml}$ amikacin for 1 hour. For CFU and MPN counts, the infected macrophages were washed three times with PBS and lysed with $0.2 \%(v / v)$ Triton X-100.

\section{Detection of Rpf peptides using mass-spectrometry}

Mtb was grown in Sauton medium to $\mathrm{OD}_{580}$ 0.8. Filtered CSN was enriched on DEAE Sepharose and digested with trypsin. LC-MS/MS was carried out using an RSLCnano HPLC system (Dionex, UK) and an LTQ-Orbitrap-los mass spectrometer (Thermo Scientific). The raw data were processed using Proteome Discoverer (version 1.4.0.288, Thermo Scientific), Mascot (version 2.2.04, Matrix Science Ltd).

\section{Statistical analyses}

Unpaired t-test (Excel) or one-way ANOVA (Prism 9) were used to analyze growth and flow cytometry datasets. Differentially expressed genes in ND- compared to CCtreated cultures, 4 hours after exposure, were identified using a modified t-test (GeneSpring 14.5; Agilent Technologies) with Benjamini and Hochberg multiple testing correction, and defined as those with $p<1 \times 10^{-5}$ and minimum fold change of 2.0.

\section{RESULTS}

\section{Design, synthesis and testing of a novel NO donor}

Exposure of mycobacteria to reactive nitrogen species lowers CFU counts [17, 28,29], which could indicate the presence of DCB. Mtb incubation with acidified nitrite in $7 \mathrm{H} 9$ medium at concentrations above $5 \mathrm{mM}$ reduced Mtb CFU counts, but resuscitation in liquid medium was not achieved (Fig. S1A). Treatment of Mtb with spermine NONOate 
(half-life of $39 \mathrm{~min}$ at $37^{\circ} \mathrm{C}, \mathrm{pH} 7.4 ;[30]$ ) did not significantly decrease CFU counts (Fig. S1A). We wondered if NO release might be too rapid for alteration of Mtb CFU counts and that sustained exposure to NO might be required for the generation of DCB. Therefore a novel NO donor (ND), 3-cyano-5-nitropyridin-2-yl diethyldithiocarbamate (Figure $1 \mathrm{~A}$ ) and a structurally related control compound (CC), 3-cyano-4,6-dimethyl-5-nitropyridin-2-yl piperidine-1-carbodithioate (Figure 1B) were designed and synthesized. The nitro group of CC was protected from hydroxyl or enzymatic attack by two methyl groups to impair release of NO in culture media. NO was progressively released from ND at $20^{\circ} \mathrm{C}, \mathrm{pH} 6.0$ with the highest concentration detected after 8 hours. NO was not produced from CC (Figure 2A).

DAF-FM diacetate can be used to detect the presence of intracellular NO [31]. DAF-FM diacetate positive $M$. bovis BCG were significantly higher $(p<0.0001)$ in NDtreated bacteria compared to CC-treated and untreated bacteria (Figure 2B). Heatkilled $M$. bovis BCG did not stain with DAF-FM diacetate after exposure to ND. This suggested that NO released from ND entered mycobacteria and was therefore suitable for testing the effects of NO exposure on growth phenotypes using $\mathrm{CC}$ as a control.

\section{Exposure to ND generated Rpf-dependent DCB}

M. bovis BCG treated with ND was stained with SYTO 9 and propidium iodide $(\mathrm{PI})$ to determine cell viability (Figure S2A). Three distinct populations were detected: unstained (PI-, SYTO 9-), dead (PI+, SYTO 9+), and live (PI-, SYTO 9+) (Figure S2A). After 24 hours there was no significant difference $(P>0.05$, t-test) in the percentage of live or dead bacteria for ND-treated or CC-treated M. bovis BCG (Figure 2C), which suggested that NO was not directly bactericidal. More than $50 \%$ of cells in both samples were PI-, SYTO 9+, and likely remained viable. 
While CC treatment for 24 hours had no effect on CFU counts (Figure S3), exposure to ND resulted in a 1000-fold reduction in CFU (Figure 3A). Furthermore $1.2 \times 10^{5}$ bacteria were recovered in $7 \mathrm{H} 9$ medium 24 hour post-treatment with ND, and supplementation with CSN increased this to $1.7 \times 10^{6}$ (Figure $3 A$ ). This suggested that the majority of the ND-treated cells were differentially culturable, in line with observations with Mtb from clinical TB samples [3, 4, 8]. Similar results were observed with $M$. bovis BCG; however, the number of resuscitated bacteria was generally lower and more variable (Figure S4).

The addition of a Rpf inhibitor [19] abolished the resuscitation of ND-treated Mtb in $7 \mathrm{H} 9$ medium, and significantly $(p<0.05)$ impaired resuscitation in $\mathrm{CSN}$ supplemented medium (Figure 3B). In accordance with previous results $[13,19]$, the Rpf- inhibitor had no effect on actively growing Mtb (Figure S5). Proteomics analysis confirmed that RpfA and RpfB were present in the Mtb CSN (Figure 3C). Together, these data suggest that Rpf proteins play an important role in resuscitation of NDgenerated DCB.

\section{Generation of Rpf-dependent Mtb by ND does not require DosR}

DosR is a response regulator that has been previously shown to respond to NO [33, 34]. Treatment of the Mtb dosR deletion mutant, a complemented strain, and the parent Mtb with ND for 24 hours resulted in similar numbers of DCB (Figure S6). All strains were equally resuscitated in CSN-supplemented medium (Figure S6), indicating that transition to the Rpf-dependent differentially culturable state after ND exposure and subsequent resuscitation by Rpf proteins did not require DosR.

\section{ND-treatment induces SigH- and SigF-transcriptional alteration and lower rpf gene expression}


Transcript profiling was used to investigate the transition of Mtb to differential culturability. Compared to treatment with CC, 640 (407 induced, 233 repressed) genes were differentially expressed in response to ND 4 hours after exposure (Figure 4A, Table S2). Analysis of up-regulated genes (using Transcription Factor Over Expression (TFOE) tool [35]) indicated this transcriptional response was coordinated by the alternative sigma factors SigH (TFOE output significance of enrichment of the regulon $2.3 \times 10^{-10}$ ) and SigF (pvalue $1.2 \times 10^{-7}$ ), both of which genes were also upregulated in response to ND (Figure $4 \mathrm{~B}$ ). SigH is activated by oxidation of its antisigma factor RshA, which can be triggered by NO, and by phosphorylation of RshA by the serine-threonine protein kinase, $\mathrm{PknB}$ [36]. SigF is associated with cell envelope integrity and is controlled by anti-sigma factor agonists, RsfA and RspB, which sequester the anti-sigma factor RsbW and also by the anti-sigma factor Rv1364c. In M. smegmatis the SigF regulon was induced by respiratory inhibitory conditions, such as might occur in the presence of $\mathrm{NO}$, in a RsfB-dependent manner [37]. The upregulation of $\operatorname{sigH}$ (3.82 fold) and $\operatorname{sigF}$ (5.79 fold) was accompanied by downregulation of the primary sigma factor, sigA (4.56-fold), and 35 genes from the $r p l, r p m$ and $r p s$ families, coding for ribosomal proteins, in accordance with impaired growth in the presence of ND (Table S2).

In contrast to other models of $\mathrm{NO}$ exposure [33, 34, 38], the DosR regulon was not induced after ND treatment (Figure 4B). Moreover, cmr, coding for a repressor of dosR expression [39], was up-regulated. Thus, the transcriptional profiling is consistent with the conclusion from our DosR deletion experiments that transition to the Rpf protein-dependent differentially culturable state after ND exposure does not require DosR . 
Mtb is regarded as an obligate aerobe using aerobic respiration to support growth and NO is an inhibitor of respiration [34]. Under stress conditions Mtb can deploy two alternative terminal electron acceptors, nitrate reductase (Nar) and fumarate reductase (Frd). In accord with the lack of induction of the DosR-regulon, narK1 was down-regulated in response to ND and expression of the narG, narH, narl, and narX genes, coding for nitrate reductase, was not significantly changed. In contrast, the frd $A B C D$ genes, coding for fumarate reductase, were up-regulated, suggesting utilization of fumarate reduction to maintain redox balance and membrane potential [40]. Interestingly, the serine-threonine protein kinase $\mathrm{G}$ (encoded by pknG), which contains a rubredoxin domain with two thioredoxin motifs and has been implicated in the regulation of intracellular $\mathrm{NADH}$ concentrations [41], was up-regulated. PknG controls central metabolism by sensing glutamate and aspartate availabilities and regulating activities of a-ketoglutarate dehydrogenase, glutamate dehydrogenase, and glutamine synthase [42]. Glutamate has been previously shown as a major carbon source for mycobacteria exposed to $\mathrm{NO}$ in vitro and during growth in macrophages [43]. In addition to these changes in operation of the Krebs cycle, the pta-ackA operon, coding for phosphotransacetylase and acetate kinase, was induced, consistent with impaired aerobic respiration and increased reliance on substrate-level phosphorylation.

Inhibition of bacterial growth by NO is linked to redox chemistry [44]. As a radical, NO itself reacts with transition metals and other molecules with unpaired electrons, such as superoxide. The latter reaction produces peroxynitrite, which can oxidize cysteine thiol to sulfenic acid. Hence, NO perturbs redox balance by damaging ironsulfur clusters and protein thiol groups [38, 45]. Accordingly, exposure to ND induced expression of anti-oxidant genes, such as the SigH/AosR-activated non-canonical 
cysteine biosynthetic genes (mec-cysO-cysM), consistent with increased synthesis of cysteine-based anti-oxidants (e.g. up-regulation of ergothioneine biosynthesis genes; rv3700c and 3704c). In addition, other thiol-related anti-oxidant activities were upregulated, including sulfate-containing compound $A B C$ transporter (cys $A 1$ ), sulfate adenyltransferase $(\operatorname{cys} D N)$, methionine sulfoxide reductases ( $m s r A$ and $m s r B$ ), a rhodanese domain protein ( $r v 1674 c)$, a thioredoxin reductase and thioredoxin proteins (trxB2-C, thiX, trxB1). Damage to iron-sulfur proteins resulting in disrupted iron homeostasis was suggested by up-regulation of mycobactin biosynthetic genes ( $m b t E, m b t G$ and $m b t H)$ and the Esx-3 genes required for siderophore-mediated iron uptake (rv0282-rv0292). These anti-oxidant responses are consistent with repair of NO-mediated oxidative damage.

The up-regulation of murl, coding for glutamate racemase [46], cw/M [47], and $p b p A$ [48] suggested that remodeling of peptidoglycan was involved in the transition to Rpf-dependency. Indeed, expression of cw/M was previously shown to be upregulated by treatment with spermine NONOate [49]. Consistent with the observation that ND-treatment resulted in Rpf-dependent mycobacteria, three rpf genes were down-regulated (rpfA, rpfB and rpfE; 9.5-, 3.9- and 33.2-fold, respectively). This was confirmed by qRT-PCR (Figure 4C).

\section{Differentially culturable mycobacteria were produced in activated THP-1 cells}

Mycobacterial pathogens can survive and replicate in macrophages where they are exposed to NO [50]. The human THP-1 monocytic cell line has been shown to produce NO after exposure to retinoic acid and THP-1 cells treated with retinoic acid and vitamin $D_{3}$ (RAVD) were proposed as a model for investigation of Mtb persistence [51].

We treated THP-1 cells with either PMA, the typical stimulant for differentiation of these cells into macrophages, or RAVD, and stained them with DAF-FM diacetate to 
detect NO (Figure 5A). The number of DAF-FM positive macrophages was significantly higher in samples treated with RAVD compared to untreated and PMAtreated cells $(p<0.05$, Figure $5 A)$.

Next, either PMA- or RAVD-derived macrophages were infected with $M$. bovis BCG. CFU and MPN counts of $M$. bovis (BCG) were determined during a 7-day infection (Figures 5B, C). In both cases, there was a significant reduction of CFU after 2 days of infection; however MPN and MPN_CSN values remained unchanged for RAVD-treated THP-1 cells. Resuscitation indices calculated for $7 \mathrm{H} 9$ medium and CSN-supplemented medium were $>1$, suggesting that at early stages of infection DCB are produced in RAVD-treated macrophages. Results obtained from PMA-derived macrophages were more variable and overall did not reveal reliable generation of DCB. Thus, there was a positive relationship between macrophage NO concentration and the generation of DCB.

\section{DISCUSSION}

During infection pathogenic mycobacteria produce heterogenous populations that differ in their metabolic activity, growth characteristics and tolerance to antimicrobials $[8,13,52]$. Amongst these populations, differentially culturable mycobacteria are of particular interest, because they are difficult to detect and eradicate, but frequently represent the dominant Mtb population in clinical TB samples [3-4, 8-12, 53-55]. In the laboratory, DCB are induced in vitro by prolonged incubation in stationary phase [56], gradual acidification [57], alteration of sodium-potassium ratio [26], treatment with antimicrobials $[14,58,59]$ or exposure to vitamin $C$ [60]. Here we describe a new model system based on a novel NO donor (ND) that simulates the in vivo environment 
by generating a heterogenous population of Mtb thereby permitting investigation of the physiologically distinct states of Mtb produced during the pathogenesis of TB.

In our ND-induced system transition to the differentially culturable state did not require activation of the DosR regulon, but involved two alternative sigma factors, SigH and SigF and their regulons, previously implicated in adaptation to redox stress and respiration-inhibitory conditions $[36,37,61]$. Overall, the transcriptomic signature of ND-treated DCB was associated with inhibition of cell division, repair of NO-induced damage, metabolic reprogramming to restore redox balance and membrane potential (Figure 6).

While there is an ongoing debate concerning the importance of NO in controlling Mtb infection in humans, NO is detected in TB patients and our data suggest that it contributes to the generation of DCB by causing the down-regulation of $r p f A, r p f B$ and rpfE expression. Rpf are cell wall cleaving enzymes and their expression under conditions when the peptidoglycan producing machinery is likely to be damaged can be fatal for mycobacteria resulting in uncontrollable cell lysis and death. In addition, NO also has the potential to directly damage Rpf proteins, which require a disulfide bond for correct folding and activity [62]. Hence the simplest explanation for the observed Rpf-dependency of ND-treated mycobacteria is that DCC cells cannot initiate Rpf production when grown in standard media and require supplementation with recombinant Rpf or Rpf-containing CSN to restart growth and peptidoglycan biosynthesis.

Expression of Rpf coding genes is tightly controlled by multiple regulators [63], Fig. S7, including Lsr2 [64] and MtrA [65]. The precise mechanism underpinning the response to ND is unknown. 
Exposure to various levels of NO drives Mtb heterogeneity in the infected host (Figure 6). These heterogenous populations have different growth requirements and respond differently to drugs, may induce differential immune responses and create a unique microbial community $(8,13)$. Our observation that $\mathrm{NO}$ down-regulates the expression of $r p f A, r p f B$ and $r p f E$ and generates Rpf-dependent DCB in vitro and intracellularly in macrophages provides the foundation for further investigations to understand the molecular mechanisms that underpin these adaptations and elucidate the precise role of DCB in TB pathogenesis and treatment outcomes.

Conflict of Interest Statement: Authors declare no conflict of interests.

Acknowledgments: We acknowledge the Centre for Core Biotechnology Services at the University of Leicester for support with Containment Level 3 experiments, flow cytometry and mass-spectrometry analyses. We would like to thank Dr Kate Gould in the Bacterial Microarray Group at St. George's University of London.

\section{REFERENCES}

1. WHO, Global tuberculosis report 2020. 2020.

2. Grace AG, Mittal A, Jain S, Tripathy JP, Satyanarayana S, Tharyan P, Kirubakaran R. Shortened treatment regimens versus the standard regimen for drug-sensitive pulmonary tuberculosis. Cochrane Database Syst Rev 2019;12:CD012918.

3. Mukamolova GV, Turapov O, Malkin J, Woltmann G, Barer MR. Resuscitationpromoting factors reveal an occult population of tubercle Bacilli in Sputum. Am J Respir Crit Care Med 2010;181:174-180.

4. Chengalroyen MD, Beukes GM, Gordhan BG, Streicher EM, Churchyard G, Hafner R, Warren R, Otwombe K, Martinson N, Kana BD. Detection and 
quantification of differentially culturable tubercle bacteria in sputum from patients with tuberculosis. Am J Respir Crit Care Med 2016;194:1532-1540.

5. Shleeva MO, Bagramyan K, Telkov MV, Mukamolova GV, Young M, Kell DB, Kaprelyants AS. Formation and resuscitation of "non-culturable" cells of Rhodococcus rhodochrous and Mycobacterium tuberculosis in prolonged stationary phase. Microbiology 2002;148:1581-1591.

6. Oliver JD. 2010. Recent findings on the viable but nonculturable state in pathogenic bacteria. FEMS Microbiol Rev 34: 415-425.

7. McAulay K, Saito K, Warrier T, Walsh KF, Mathurin LD, Royal-Mardi G, Lee MH, Ocheretina O, Pape JW, Fitzgerald DW, Nathan CF. Differentially detectable Mycobacterium tuberculosis cells in sputum from treatment-naive subjects in Haiti and their proportionate increase after initiation of treatment. mBio 2018;9.

8. Turapov O, O'Connor BD, Sarybaeva AA, Williams C, Patel H, Kadyrov AS, Sarybaev AS, Woltmann G, Barer MR, Mukamolova GV. Phenotypically adapted Mycobacterium tuberculosis populations from sputum are tolerant to first line drugs. Antimicrob Agents Chemother 2016;60:2476-2483.

9. Beltran CGG, Gallant J, Venter R, du Plessis N, Loxton AG, Trost M, Winter J, Malherbe ST, Kana BD, WalzI G. Investigating non-sterilizing cure in TB patients at the end of successful anti-TB therapy. Front Cell Infect Microbiol 2020;10:443.

10. Zainabadi K, Walsh KF, Vilbrun SC, Mathurin LD, Lee MH, Saito K, Mishra S, Ocheretina O, Pape JW, Nathan C et al. Characterization of differentially detectable Mycobacterium tuberculosis in the sputum of subjects with drug sensitive or drug resistant tuberculosis before and after two months of therapy. Antimicrob Agents Chemother 2021:AAC0060821. 
11. Rosser A, Stover C, Pareek M, Mukamolova GV. Resuscitation-promoting factors are important determinants of the pathophysiology in Mycobacterium tuberculosis infection. Crit Rev Microbiol 2017;43:621-630.

12. Huang W, Qi Y, Diao Y, Yang F, Zha X, Ren C, Huang D, Franken KL, Ottenhoff $\mathrm{TH}$, Wu Q, Shen J. Use of resuscitation-promoting factor proteins improves the sensitivity of culture-based tuberculosis testing in special samples. Am J Respir Crit Care Med 2014;189:612-614.

13. Turapov O, Glenn S, Kana B, Makarov V, Andrew PW, Mukamolova GV. The in vivo environment accelerates generation of resuscitation-promoting factordependent mycobacteria. Am J Respir Crit Care Med 2014;190:1455-1457.

14. Hu Y, Liu A, Ortega-Muro F, Alameda-Martin L, Mitchison D, Coates A. Highdose rifampicin kills persisters, shortens treatment duration, and reduces relapse rate in vitro and in vivo. Front Microbiol 2015;6: 641.

15. Forstermann U, Sessa WC. Nitric oxide synthases: regulation and function. Eur Heart J 2012;33:829-837, 837a-837d.

16. MacMicking JD, North RJ, LaCourse R, Mudgett JS, Shah SK, Nathan CF. Identification of nitric oxide synthase as a protective locus against tuberculosis. Proc Natl Acad Sci U S A 1997;94:5243-5248.

17. Jamaati H, Mortaz E, Pajouhi Z, Folkerts G, Movassaghi M, Moloudizargari M, Adcock IM, Garssen J. Nitric Oxide in the pathogenesis and treatment of tuberculosis. Front Microbiol 2017;8: 2008.

18. Mishra BB, Lovewell RR, Olive AJ, Zhang G, Wang W, Eugenin E, Smith CM, Phuah JY, Long JE, Dubuke ML et al. Nitric oxide prevents a pathogenpermissive granulocytic inflammation during tuberculosis. Nat Microbiol $2017 ; 2: 17072$. 
19. Demina GR, Makarov VA, Nikitushkin VD, Ryabova OB, Vostroknutova GN, Salina EG, Shleeva MO, Goncharenko AV, Kaprelyants AS. Finding of the low molecular weight inhibitors of resuscitation promoting factor enzymatic and resuscitation activity. PLoS One, 2009;4:e8174.

20. Parish T, Smith DA, Kendall S, Casali N, Bancroft GJ, Stoker NG. Deletion of two-component regulatory systems increases the virulence of Mycobacterium tuberculosis. Infect Immun, 2003, 71: 1134-40.

21. Jarvis B, Wilrich $\mathrm{C}$, Wilrich PT. Reconsideration of the derivation of Most Probable Numbers, their standard deviations, confidence bounds and rarity values. J Appl Microbiol 2010;109:1660-1667.

22. Verdon CP, Burton BA, Prior RL. Sample pretreatment with nitrate reductase and glucose-6-phosphate dehydrogenase quantitatively reduces nitrate while avoiding interference by $\mathrm{NADP}^{+}$when the Griess reaction is used to assay for nitrite. Anal Biochem, 1995;224:502-508.

23. Sheng JZ, Wang D, Braun AP. DAF-FM (4-amino-5-methylamino-2',7'difluorofluorescein) diacetate detects impairment of agonist-stimulated nitric oxide synthesis by elevated glucose in human vascular endothelial cells:reversal by vitamin C and L-sepiapterin. J Pharmacol Exp Ther 2005;315: 931-940.

24. Simeone R, Bobard A, Lippmann J, Bitter W, Majlessi L, Brosch R, Enninga J. Phagosomal rupture by Mycobacterium tuberculosis results in toxicity and host cell death. PLoS Pathog, 2012;8:e1002507.

25. Waddell SJ, Butcher PD. Use of DNA arrays to study transcriptional responses to antimycobacterial compounds. I. In Antibiotic Resistance Protocols (SH Gillespie and TD McHugh Eds.) Methods Mol Biol 2010;642:75-91.

26. Salina EG, Waddell SJ, Hoffmann N, Rosenkrands I, Butcher PD, 
Kaprelyants AS. Potassium availability triggers Mycobacterium tuberculosis transition to, and resuscitation from, non-culturable (dormant) states. Open Biol 2014;4:140106.

27. Rachman H, Lee JS, Angermann J, Kowall J, Kaufmann SH. Reliable amplification method for bacterial RNA. J Biotechnol, 2006;126:61-68.

28. O'Brien L, Carmichael J, Lowrie DB, Andrew PW. Strains of Mycobacterium tuberculosis differ in susceptibility to reactive nitrogen intermediates in vitro. Infect Immun 1994;62:5187-190.

29. Gample SP, Agrawal S, Sarkar D. Evidence of nitrite acting as a stable and robust inducer of non-cultivability in Mycobacterium tuberculosis with physiological relevance. Sci Rep 2019;9:9261.

30. Li B, Ming Y, Liu Y, Xing H, Fu R, Li Z, Ni R, Li L, Duan D, Xu J, Li C et al. Recent Developments in Pharmacological Effect, Mechanism and Application Prospect of Diazeniumdiolates. Front Pharmacol 2020;11:923.

31. Sheng JZ, Wang D, Braun AP. DAF-FM (4-amino-5-methylamino-2',7'difluorofluorescein) diacetate detects impairment of agonist-stimulated nitric oxide synthesis by elevated glucose in human vascular endothelial cells:reversal by vitamin C and L-sepiapterin. J Pharmacol Exp Ther 2005;315:931-940.

33. Kendall SL, Movahedzadeh F, Rison SC, Wernisch L, Parish T, Duncan K, Betts JC, Stoker NG. The Mycobacterium tuberculosis dosRS two-component system is induced by multiple stresses. Tuberculosis (Edinb) 2004;84:247-255.

34. Voskuil MI, Schnappinger D, Visconti KC, Harrell MI, Dolganov GM, Sherman DR, Schoolnik GK. Inhibition of respiration by nitric oxide induces a Mycobacterium tuberculosis dormancy program. J Exp Med 2003;198:705-713. 
35. Rustad TR, Minch KJ, Ma S, Winkler JK, Hobbs S, Hickey M, Brabant W, Turkarslan S, Price ND, Baliga NS, Sherman DR. Mapping and manipulating the Mycobacterium tuberculosis transcriptome using a transcription factor overexpression-derived regulatory network. Genome Biol 2014;15:502.

36. Park ST, Kang CM, Husson RN. Regulation of the SigH stress response regulon by an essential protein kinase in Mycobacterium tuberculosis. Proc Natl Acad Sci U S A 2008;105:13105-13110.

37. Oh Y, Song SY, Kim HJ, Han G, Hwang J, Kang HY, Oh Jl. The partner switching system of the SigF sigma factor in Mycobacterium smegmatis and Induction of the SigF regulon under respiration-inhibitory conditions. Front Microbiol $2020 ; 11: 588487$.

38. Cortes T, Schubert OT, Banaei-Esfahani A, Collins BC, Aebersold R,

Young DB. Delayed effects of transcriptional responses in Mycobacterium tuberculosis exposed to nitric oxide suggest other mechanisms involved in survival. Sci Rep 2017;7:8208.

39. Smith LJ, Bochkareva A, Rolfe MD, Hunt DM, Kahramanoglou C, Braun Y, Rodgers A, Blockley A, Coade S, Lougheed KEA, Hafneh NA. et al. Cmr is a redox-responsive regulator of DosR that contributes to $M$. tuberculosis virulence. Nucleic Acids Res, 2017;45:6600-6612.

40. Watanabe S, Zimmermann M, Goodwin MB, Sauer U, Barry CE, Boshoff HI. Fumarate reductase activity maintains an energized membrane in anaerobic Mycobacterium tuberculosis. PLoS Pathog 2011;7:e1002287.

41. Wolff KA, de la Pena A H, Nguyen HT, Pham TH, Amzel LM, Gabelli SB, Nguyen L. A redox regulatory system critical for mycobacterial survival in macrophages and biofilm development. PLoS Pathog 2015;11:e1004839. 
42. Rieck B, Degiacomi G, Zimmermann M, Cascioferro A, Boldrin F, Lazar-Adler NR, Bottrill AR, le Chevalier F, Frigui W, Bellinzoni M et al. PknG senses amino acid availability to control metabolism and virulence of Mycobacterium tuberculosis. PLoS Pathog 2017;13:e1006399.

43. Gallant JL, Viljoen AJ, van Helden PD, Wiid IJ. Glutamate dehydrogenase Is required by Mycobacterium bovis BCG for resistance to cellular stress. PLoS One 2016;11:e0147706.

44. Lancaster JR. Nitric oxide: a brief overview of chemical and physical properties relevant to therapeutic applications. Future Sci OA 2015;1: FSO59.

45. Crack JC, Green J, Thomson AJ, Le Brun NE. Iron-sulfur clusters as biological sensors:the chemistry of reactions with molecular oxygen and nitric oxide. Acc Chem Res 2014;47:3196-205.

46. Maitra A, Munshi T, Healy J, Martin LT, Vollmer W, Keep NH, Bhakta S. Cell wall peptidoglycan in Mycobacterium tuberculosis: An Achilles' heel for the TBcausing pathogen. FEMS Microbiol Rev 2019;43:548-575.

47. Turapov O, Forti F, Kadhim B, Ghisotti D, Sassine J, Straatman-Iwanowska A, Bottrill AR, Moynihan PJ, Wallis R, Barthe P et al. Two Faces of CwIM, an Essential PknB Substrate, in Mycobacterium tuberculosis. Cell Rep 2018;25:5767 e5.

48. Arora D, Chawla Y, Malakar B, Singh A, Nandicoori VK. The transpeptidase $\mathrm{PbpA}$ and noncanonical transglycosylase RodA of Mycobacterium tuberculosis play important roles in regulating bacterial cell lengths. J Biol Chem 2018; 293: $6497-6516$. 
49. Bancroft PJ, Turapov O, Jagatia H, Arnvig KB., Mukamolova GV, Green J. Coupling of peptidoglycan synthesis to central metabolism in mycobacteria: post-transcriptional control of CwIM by aconitase. Cell Rep 2020;32:108209.

50. Braverman J, Stanley SA. Nitric Oxide modulates macrophage responses to Mycobacterium tuberculosis infection through activation of HIF-1alpha and repression of NF-kappaB. J Immunol 2017. 199:1805-1816.

51. Estrella JL, Kan-Sutton C, Gong X, Rajagopalan M, Lewis DE, Hunter RL, Eissa NT, Jagannath C. A novel in vitro human macrophage model to study the persistence of Mycobacterium tuberculosis using vitamin $\mathrm{D}(3)$ and retinoic acid activated THP-1 macrophages. Front Microbiol 2011;2:67.

52. Manina G, Dhar N, McKinney JD. Stress and host immunity amplify Mycobacterium tuberculosis phenotypic heterogeneity and induce nongrowing metabolically active forms. Cell Host Microbe 2015;17:32-46.

53. Dhillon J, Fourie PB, Mitchison DA. Persister populations of Mycobacterium tuberculosis in sputum that grow in liquid but not on solid culture media. $J$ Antimicrob Chemother 2014;69:437-440.

54. Rosser A, Pareek M, Turapov O, Wiselka MJ, Mukamolova GV. Differentially culturable tubercule bacilli are generated during non-pulmonary tuberculosis infection. Am J Respir Crit Care Med 2018;197:818-821.

55. Dusthackeer A, Balasubramanian M, Shanmugam G, Priya S, Nirmal CR, Sam Ebenezer R, Balasubramanian A, Mondal RK, Thiruvenkadam K, Hemanth Kumar AK, Ramachandran G, Subbian S. Differential culturability of Mycobacterium tuberculosis in culture-negative sputum of patients with pulmonary tuberculosis and in a simulated model of dormancy. Front Microbiol $2019 ; 10: 2381$. 
56. Shleeva MO, Bagramyan K, Telkov MV, Mukamolova GV, Young M, Kell DB, Kaprelyants AS. Formation and resuscitation of "non-culturable" cells of Rhodococcus rhodochrous and Mycobacterium tuberculosis in prolonged stationary phase. Microbiology 2002;148:1581-1591.

57. Shleeva MO, Kudykina YK, Vostroknutova GN, Suzina NE, Mulyukin AL, Kaprelyants AS. Dormant ovoid cells of Mycobacterium tuberculosis are formed in response to gradual external acidification. Tuberculosis (Edinb), 2011;91:146154.

58. Loraine J, Pu F, Turapov O, Mukamolova GV. Development of an in vitro assay for detection of drug-Induced Resuscitation-promoting-factor-dependent mycobacteria. Antimicrob Agents Chemother 2016;60:6227-33.

59. Saito K, Warrier T, Somersan-Karakaya S, Kaminski L, Mi J, Jiang X, Park S, Shigyo K, Gold B, Roberts J et al. Rifamycin action on RNA polymerase in antibiotic-tolerant Mycobacterium tuberculosis results in differentially detectable populations. Proc Natl Acad Sci U S A 2017;114:E4832-E4840.

60. Taneja NK, Dhingra S, Mittal A, Naresh M, Tyagi JS. Mycobacterium tuberculosis transcriptional adaptation, growth arrest and dormancy phenotype development is triggered by vitamin C. PLoS One 2010;5:e10860.

61. Manganelli R, Voskuil MI, Schoolnik GK, Dubnau E, Gomez M, Smith I. Role of the extracytoplasmic-function sigma factor $\sigma^{\mathrm{H}}$ in Mycobacterium tuberculosis global gene expression. Mol Microbiol 2002;45:365-374.

62. Cohen-Gonsaud M, Barthe P, Bagneris C, Henderson B, Ward J, Roumestand C, Keep NH. The structure of a resuscitation-promoting factor domain from Mycobacterium tuberculosis shows homology to lysozymes. Nat Struct Mol Biol 2005;12:270-273. 
63. Schwenk S, Arnvig KB. Regulatory RNA in Mycobacterium tuberculosis, back to basics. Pathog Dis 2018;76:fty035.

64. Bartek IL, Woolhiser LK, Baughn AD, Basaraba RJ, Jacobs WR, Lenaerts AJ, Voskuil MI. Mycobacterium tuberculosis Lsr2 is a global transcriptional regulator required for adaptation to changing oxygen levels and virulence. mBio 2014;5:e01106-14.

65. Singh KK, Athira PJ, Bhardwaj N, Singh DP, Watson U, Saini DK. Acetylation of response regulator protein MtrA in M. tuberculosis regulates its repressor activity. Front Microbiol 2020;11:516315.

66. Balazsi G, Heath AP, Shi L, Gennaro ML. The temporal response of the Mycobacterium tuberculosis gene regulatory network during growth arrest. $\mathrm{Mol}$ Syst Biol 2008;4:225.

67. Cole ST, Brosch R, Parkhill J, Garnier T, Churcher C, Harris D, Gordon SV, Eiglmeier K, Gas S, Barry CE, et al. Deciphering the biology of Mycobacterium tuberculosis from the complete genome sequence. Nature 1998;393:537-544. 


\section{FIGURE LEGENDS}

Figure 1. Chemical structures of nitric oxide donor (ND) and a control compound (CC) used in this study. (A) The NO donor, 3-cyano-5-nitropyridin-2-yl diethyldithiocarbamate 3-cyano-4 (ND). (B) The control compound, 6-dimethyl-5nitropyridin-2-yl piperidine-1-carbodithioate (CC).

Figure 2. (A) Quantifying NO release from ND and CC. Both compounds were incubated in sodium phosphate buffer for 24 hours and the amount of NO released was measured using Griess reagent. (B) Monitoring NO release from ND or CC after treatment of $M$. bovis BCG for 24 hours using DAF-FM diacetate and flow cytometry. The percentage of DAF-FM diacetate stained shown as mean \pm SEM $(N=3)$. (C) Assessing the viability of $M$. bovis BCG following treatment with ND and CC. Bacteria were dually stained with PI and SYTO 9 at 24 hours post-treatment with either ND or CC and analyzed by flow cytometry. Percentages of live (SYTO 9+), dead (SYTO 9+ $\mathrm{PI}+)$, or unstained bacteria are shown as mean $\pm \mathrm{SEM}(\mathrm{N}=3)$.

Figure 3. Generation of Rpf-dependent Mtb in vitro. (A) Mtb were treated with $100 \mu \mathrm{M}$ $\mathrm{NO}$ donor (ND) for 24 hours at $37^{\circ} \mathrm{C}$ without shaking. CFU, MPN and MPN_CSN counts were determined before treatment and after 24 hours. (B) The effect of Rpfinhibitors on the resuscitation of ND-treated mycobacteria. Rpf-inhibitor (3-nitro-4thiocyanato-phenyl)-phenyl-methanone) $35 \mu \mathrm{M}$ was added to both $7 \mathrm{H} 9$ medium (7H9 plus Rpf-inhibitor) and 7H9 supplemented with CSN (CSN plus Rpf-inhibitor). Results are the mean $\pm \operatorname{SEM}(n=3)$. Resuscitation Index $=\log _{10}$ MPN - $\log _{10}$ CFU. (C) Detection of RpfA and RpfB peptides in Mtb CSN by mass-spectrometry.

Figure 4. Exposure of Mtb to ND for 4 hours results in activation of distinct regulatory mechanisms. (A) Volcano plot showing 407 genes significantly induced (red) and 233 genes repressed (blue) by ND compared to CC at 4 hours. Significantly differentially 
expressed genes were identified using a moderated t-test $\left(p<1 \times 10^{-5}\right.$ with Benjamini and Hochberg multiple testing correction) and fold change >2.0. (B) Box and whisker plots showing the distribution of expression ratios (log2) of the SigH regulon [66], aerobic respiration genes functional classification I.B.6.a [67] and DosR regulon [66] after 4 hours exposure to ND or CC. (C) Expression of selected genes was confirmed by qPCR from Mtb exposed to ND relative to CC at 4 hours normalised to $16 \mathrm{~S}$ rRNA transcript abundance. Data shown as the $\log _{2}$ (relative fold change) $\pm \operatorname{SEM}(N=3)$.

Figure 5. THP-1 macrophages generate Rpf-dependent M. bovis BCG. (A) NO levels in PMA-activated and RAVD-activated THP-1 cells detected using DAF-FM diacetate and flow cytometry. The percentages of DAF-FM diacetate-stained cells are shown as the mean+/-SEM. * $p<0.05$. RAVD-treated $(B, C)$ or PMA-treated $(D, E)$ THP-1 cells infected with $M$. bovis BCG at MOI 0.5. CFU, MPN and MPN_CSN counts were determined at $0,2,4$ and 7 days post-infection. ${ }^{* *} \mathrm{CFU}$ counts were significantly lower than MPN counts $(p<0.01) ;{ }^{* *}$ CFU counts were significantly lower than MPN_CSN counts $(p<0.001)$. The $\mathrm{RI}\left(\mathrm{RI}=\log _{10}(\mathrm{MPN} / \mathrm{ml})-\log _{10}(\mathrm{CFU} / \mathrm{ml})\right)$ were calculated for both PMA-activated (C) and RAVD-activated THP-1 cells (E). (C) *RI_7H9 and RI_CSN at 2 days were significantly higher those at the start of infection $(p<0.05)$.

Figure 6. NO-mediated generation of Rpf-dependent differentially culturable Mtb. NO exposure in vitro induces global transcriptional changes coordinated by SigH and SigF which result in growth shut-down, activation of alternative metabolic pathways, detoxification of $\mathrm{NO}$ damage and down-regulation of $r p f A$, rpfB and rpfE expression. This leads to generation of a heterogenous population of Mtb, including a Rpfdependent sub-population. This population heterogeneity mimics in vivo observations where NO is produced by the inducible NO synthase (iNOS) and drives formation of 
bioRxiv preprint doi: https://doi.org/10.1101/2021.09.28.462152; this version posted September 28, 2021. The copyright holder for this preprint (which was not certified by peer review) is the author/funder, who has granted bioRxiv a license to display the preprint in perpetuity. It is made available under aCC-BY 4.0 International license.

Rpf-dependent and differentially culturable Mtb that can be detected in infected tissue and sputum samples. 


\section{FIGURES}

A.<smiles>CCNC(=S)Sc1ncc([N+](=O)[O-])cc1C#N</smiles>

B.

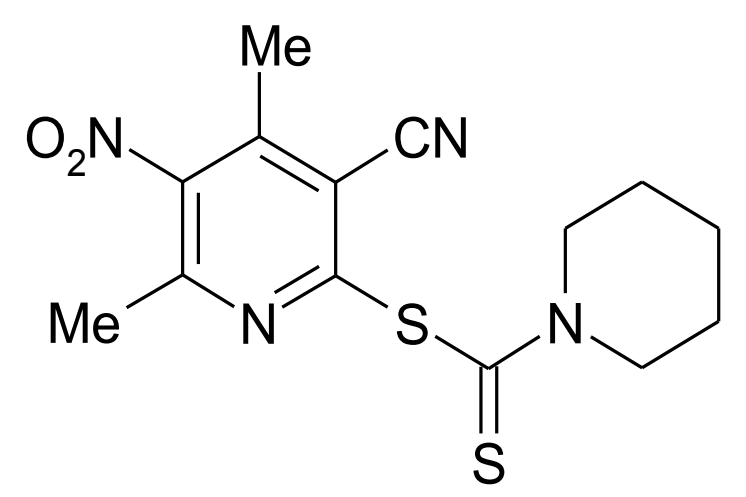

\section{FIGURE 1.}




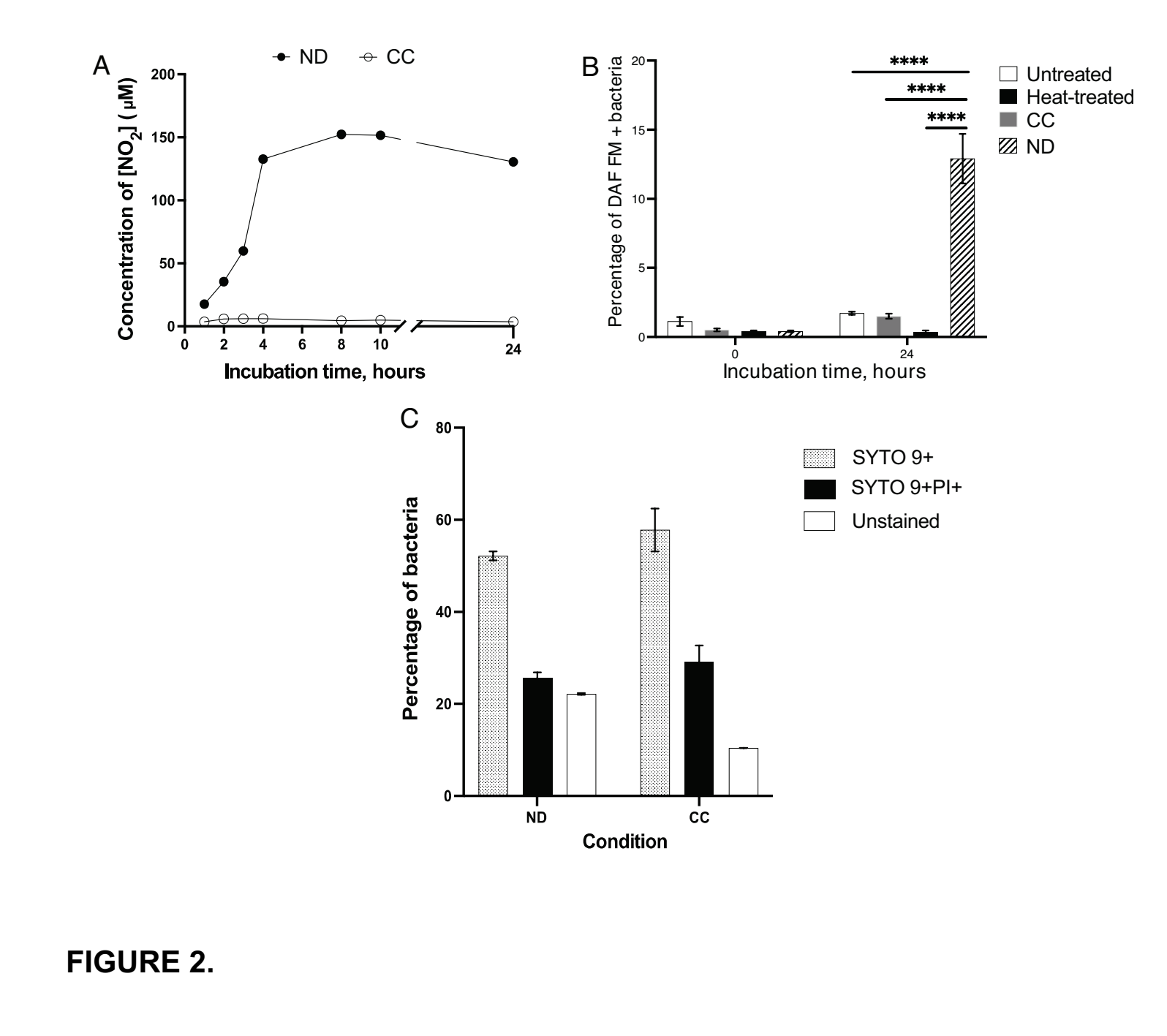



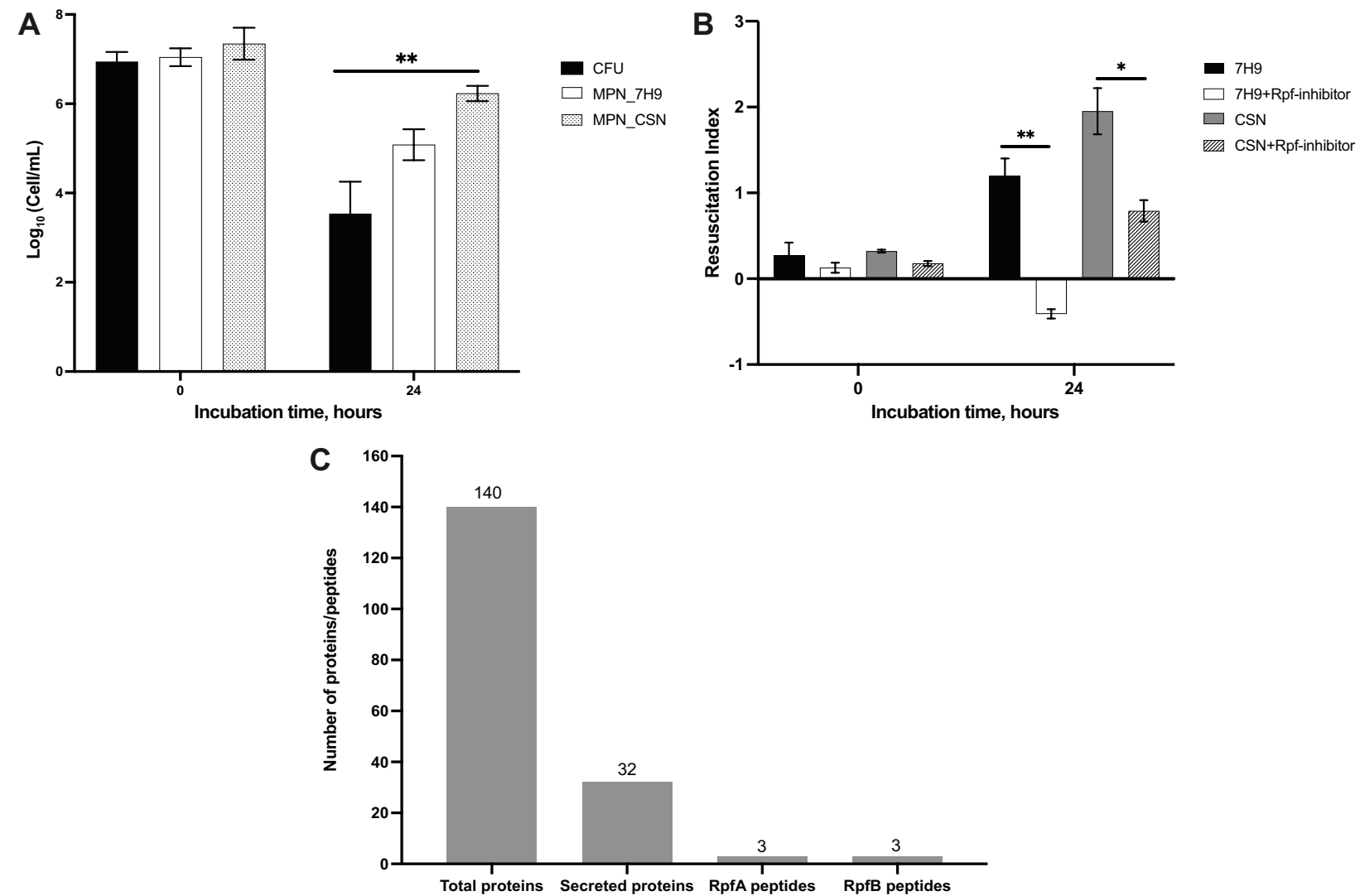

FIGURE 3. 

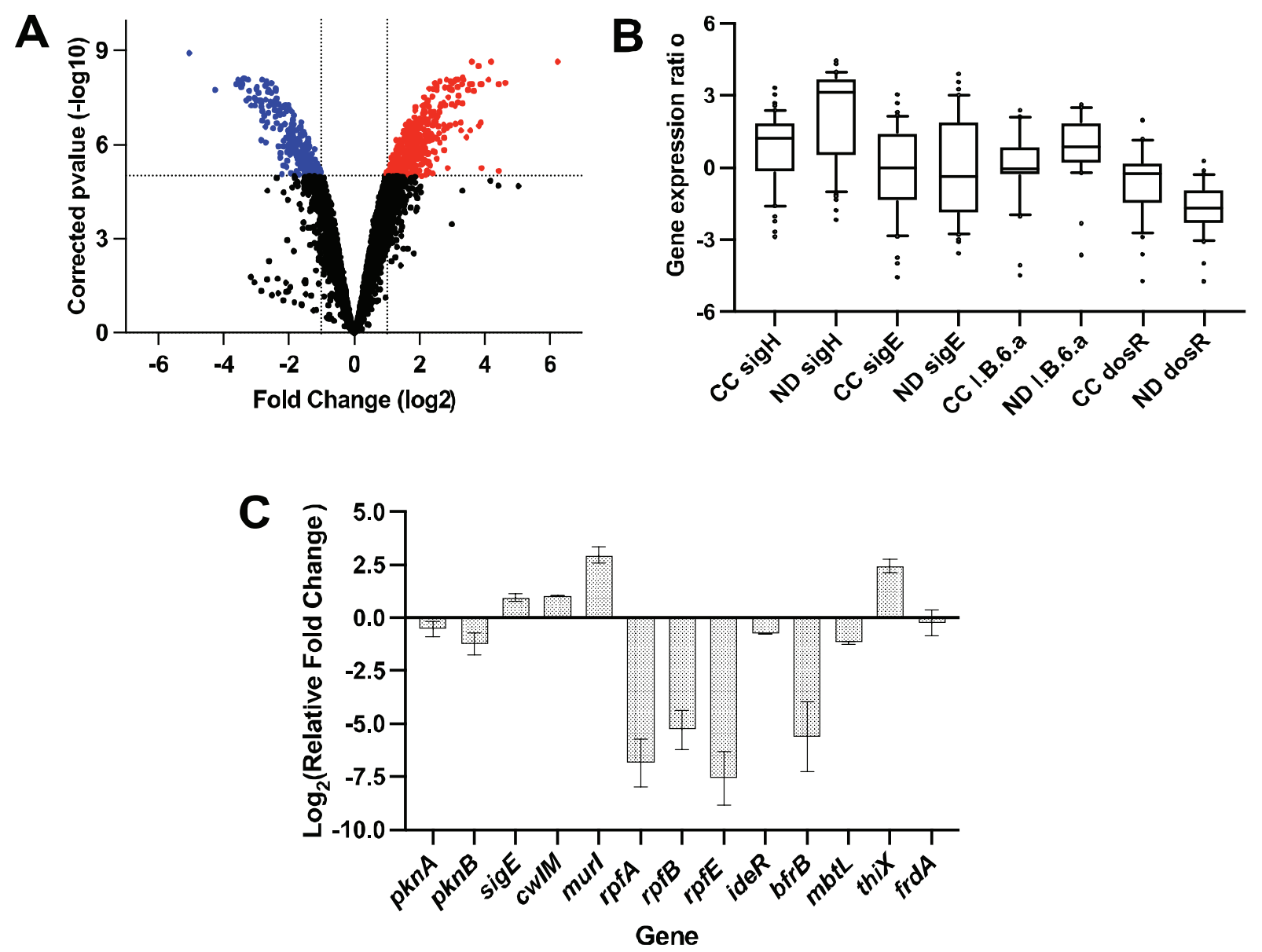

FIGURE 4. 

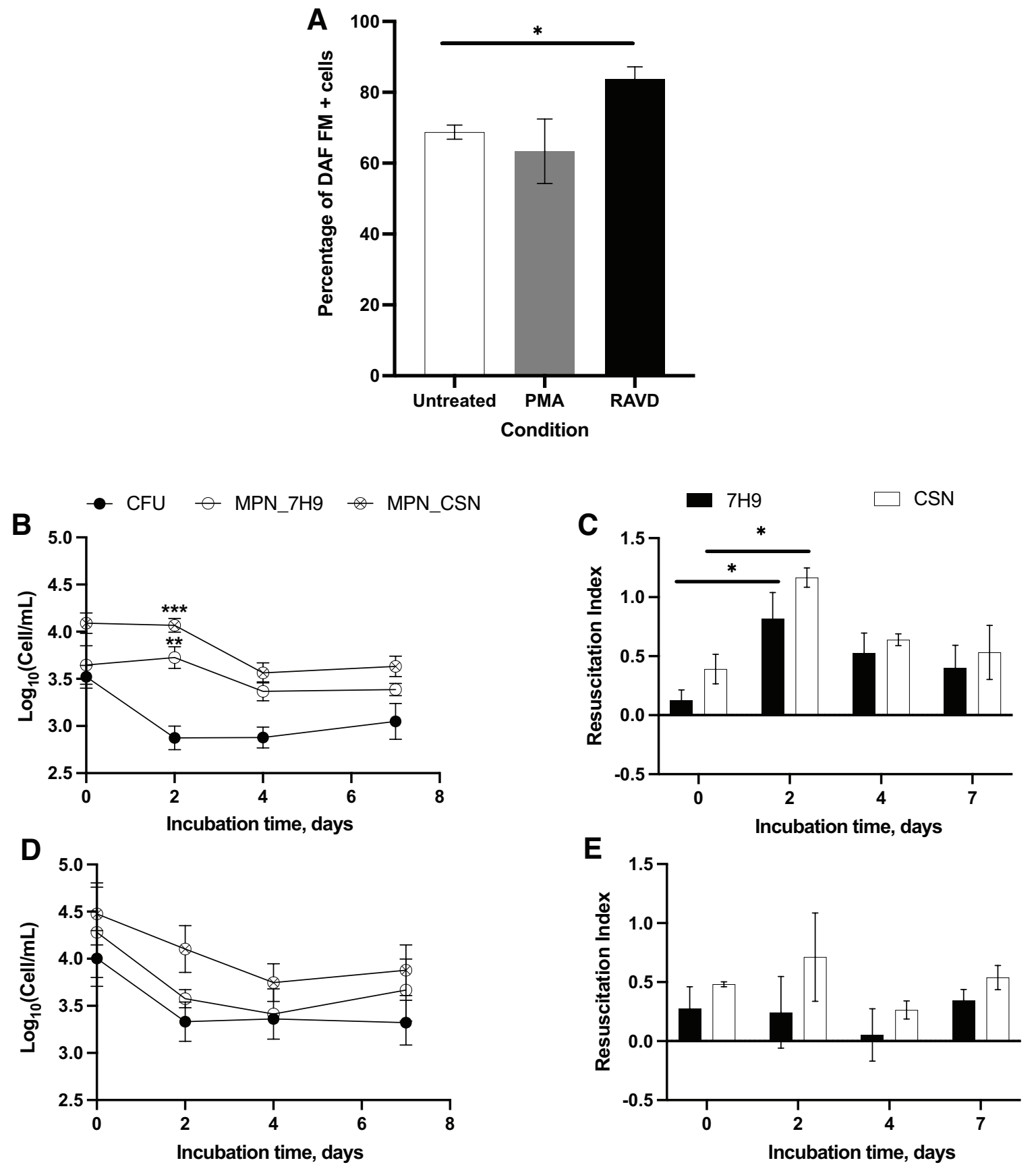

FIGURE 5. 


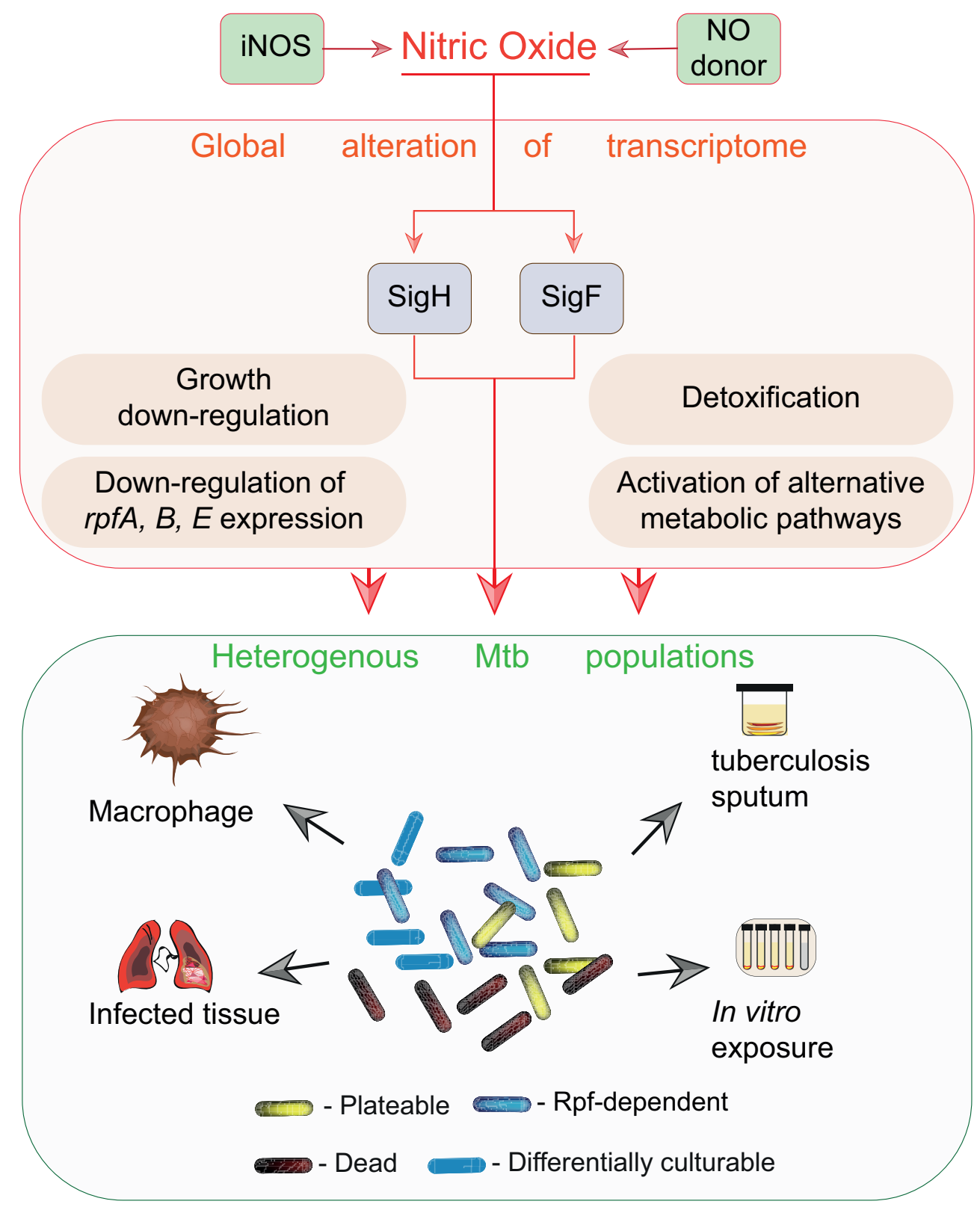

FIGURE 6. 